\title{
Preliminary Assessment of Goat Piroplasmosis in Benadir Region, Somalia
}

\author{
Ahmed Abdulkadir Hassan ${ }^{1}$, Abdalla Mohamed Ibrahim ${ }^{2}$, \\ Rabab Haroon Mohamed ${ }^{2}$, Hussein Haji Aden ${ }^{3}$ \\ ${ }^{1}$ Jazeera Quarantine, Mogadishu, Somalia \\ ${ }^{2}$ College of Veterinary Medicine, University of Bahri, Khartoum, Sudan \\ ${ }^{3}$ Somali Animal Health Service Project (SAHSP), Mogadishu, Somalia \\ Email: kadle010@gmail.com
}

Received July 30, 2013; revised August 30, 2013; accepted September 10, 2013

Copyright (C) 2013 Ahmed Abdulkadir Hassan et al. This is an open access article distributed under the Creative Commons Attribution License, which permits unrestricted use, distribution, and reproduction in any medium, provided the original work is properly cited.

\begin{abstract}
Haemoparasites are major-constraints on livestock production in tropical and sub-tropical countries. This study was conducted during 2012-2013 to determine the prevalence of blood parasites in goats of small-holders in Benadir region, Somalia and update epidemiological data that had already lost during the civil war in the country. A total of 100 blood samples were collected from goat in Wadajir (47 goat) and Dharkeynley (53 goat) districts, using venipuncture of jugular vein. The samples were examined for the presence of blood parasites using light microscopy. Some ticks when presented in the sampled animals-were also collected for tick identification. Analysis of blood smears revealed $100 \%$ samples positive for blood parasites. Out of these cases, 22 samples (22\%) were harboring single infection of Babesia spp. and 14 samples (14\%) were having single infection of Theileria spp. Interestingly the Remaining 64 blood samples (64\%) showed mixed infection of Babesia spp. with Theileria spp. Rhipicephalus evertsi (72.84\%), Rhipicephalus pulchellus (34.57\%), Amblyomma lepidum (3.70\%) and Hyalomma rufipes (1.23\%) were identified from the investigated goats. In conclusion, the findings of this study indicated that, the prevalence of tick and tick-borne diseases were considered to be high in Benadir region of Somalia. A further area wide in-depth study is recommended in the country.
\end{abstract}

Keywords: Piroplasmosis; T\&TBDs; Goat; Benadir Region; Somalia

\section{Introduction}

Haemoprotozoan parasites are the main livestock production constraints all over the world ([1-3]). Causing serious economical losses, tick and tick borne diseases ( $\mathrm{T}$ \& TBDs) still remain to be a major threat to animals in tropical and sub tropical countries $([1,4,5])$ including Somalia. In case of these blood parasites infection up to $75 \%$ erythrocytes may be destroyed in fatal cases and even in milder infection so many erythrocytes are destroyed, then a severe anaemia result ([1,2]). Babesiosis, Theileriosis and Ehrelichiosis (Cowdriosis and Anaplasmosis) are the major TBDs that cause serious diseases among Central and East African animals including goats $([1,4])$. This study was undertaken to know the ubiquity of T\&TBDs prevalent in goat in Benadir region, Somalia. This will further pave the way for launching sustainable animal disease controlling/minimizing in Somalia. The livestock sector in Somalia is an important contributor to the overall economy of Somalia, with its vast rangeland grazing area and large animal population. They are adapted to a nomadic way of grazing which may be migrated through borders with Djibouti, Ethiopia and Kenya. This may affect the distribution of such diseases in the country. There is little information on national herd distribution and composition up to date. About 37.5 millions grazing animals were reported $([6,7])$. Other data gathered by the Food Security Assessment Unit [8] were a total of 38.9 millions grazing animals. The compsition of animals in southern Somalia is shown in the Table 1.

The total collapse of the state and lawlessness resulted in the loss of most animal health services and facilitates scarcity of research projects. Then the spread of livestock diseases in the country is uncontrolled. Therefore this study will contribute on a recent data base of parasitical diseases in Somalia. 


\section{Materials and Methods}

\section{Study Area:}

Before the outbreak of the civil war in 1991, the Democratic Republic of Somalia covered an area of 638,000 square kilometres in the Horn of Africa. Somalia's landmass is dominated by arid and semiarid rangelands for which pastoralism is the most appropriate form of land use.

Benadir region is one the southern regions of Somalia. The Districts of study are Wadajir and Dharkeynley which commonly known as "Medina" (See Figure 1).

Animals: Goats of small-holders in Benadir region, Somalia.

Samples:

Blood samples: A total of 100 goat blood samples were collected aseptically in a sterilized syringe from the jugular vein and transferred into blood containers containing EDTA. A drop of blood was also spotted on filter paper, dried and stored in $-20^{\circ} \mathrm{C}$ until needed for further molecular confirmation. Thin blood smears were prepared and fixed with absolute methanol and stained with Giemsa's stain. These samples were transported to Sudan for parasitological investigation. The stained blood smears were examined microscopically in Parasitology Lab., College of Veterinary Medicine, Sudan University of Science and Technology (SUST).

Ticks samples: Ticks were collected from some of the sampled animals and preserved in $70 \%$ ethanol and transported to Sudan for tick identification. These samples were identified in the Department of Entomology

Table 1. Livestock population in Southern Somalia [8].

\begin{tabular}{|c|c|c|c|c|c|}
\hline Area & Camels & Cattle & Sheep & Goats & Total \\
\hline Southern Somalia & $1,217,470$ & $1,340,870$ & 707,020 & $1,860,110$ & $5,125,470$ \\
\hline
\end{tabular}

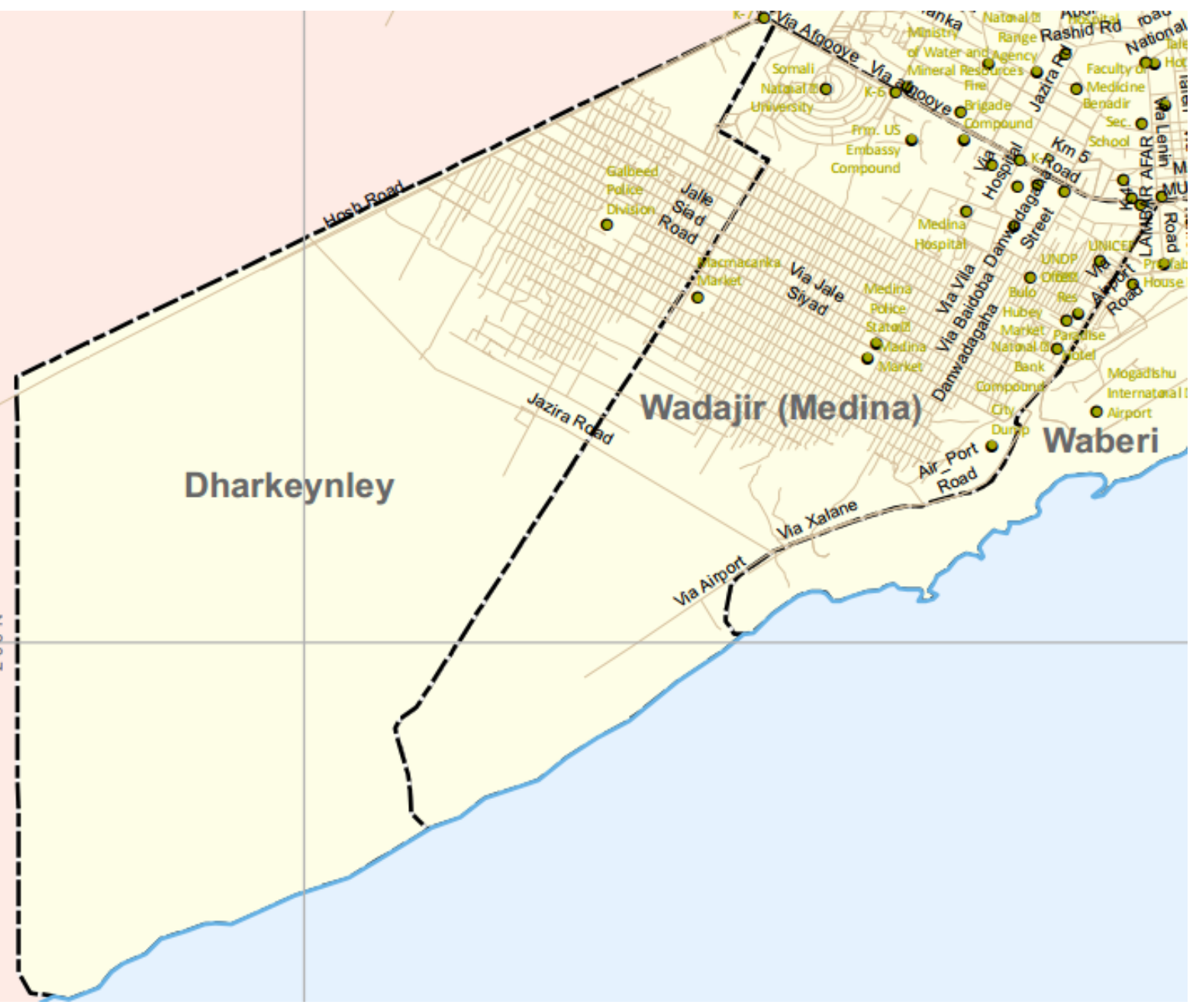

Figure 1. Wadajir and Dharkeynley districts. 
and Acarology, Veterinary Research Institute (VRI), Khartoum, Sudan.

\section{Results and Discussion}

In this study, screening test of 100 blood samples reveals that all blood smears were positive with hyperparasitaemia of Piroplasms (Plate 1). Despite the animal is apparently healthy and this might be due to the unusual acaricidal practice among goat householders or due to host resistant to these parasites, hence there is a hyperparasitaemia as well as mixed infection of Piroplasms. A further investigation using advanced molecular techniques is needed in this aspect.

As shown in Table 2, analysis of stained blood smears revealed $100 \%$ samples positive for blood parasites. Out of these cases, 22 samples $(22 \%)$ were harboring single infection of Babesia spp. and 14 samples (14\%) were having single infection of Theileria spp. Interestingly the remaining 64 blood samples (64\%) showed mixed infection of Babesia spp. with Theileria spp.

In the present study Rhipicephalus evertsi is found to be the superior $(72.84 \%)$ among goat ticks in Somalia (Plate 2), followed by Rhipicephalus pulchellus (34.57\%), Amblyomma lepidum (03.70\%) and Hyalomma rufipes $(01.23 \%)$. This may reflect the high prevalence of TBDs reported in these goats. Similar tick species were identified in East Africa [1].

\section{Acknowledgements}

We are grateful to acknowledge Abdullah Abukar Hassan (Afgooye) for his dynamic guidance and sympathetic attitude during this study. We would like to thank Prof.

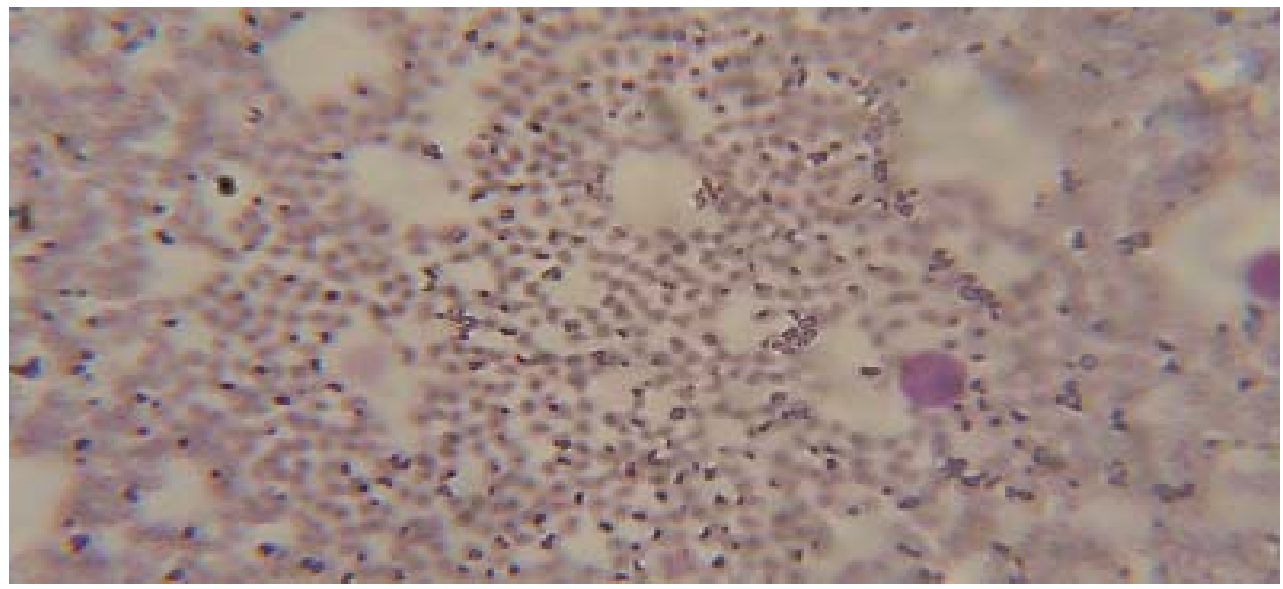

Plate 1. Somali goat blood smear showing hyper-parasitaemia of Piroplasms.

Table 2. Analysis of stained blood smears.

\begin{tabular}{|c|c|c|c|}
\hline Piroplasms Area & Dharkeynley $(\mathrm{n}=53)$ & Wadajir $(n=47)$ & Total Prevalence \\
\hline Babesia spp. & $7(13.21 \%)$ & $15(31.91 \%)$ & $22(22 \%)$ \\
\hline Theileria spp. & $9(16.98 \%)$ & $5(10.64 \%)$ & $14(14 \%)$ \\
\hline Mix infection & $37(69.81 \%)$ & $27(57.45 \%)$ & $64(64 \%)$ \\
\hline Overall prevalence & $53(100 \%)$ & $47(100 \%)$ & $100(100 \%)$ \\
\hline
\end{tabular}

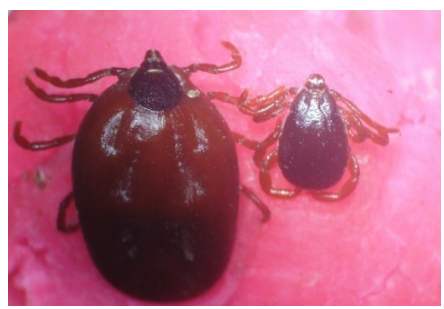

(a)

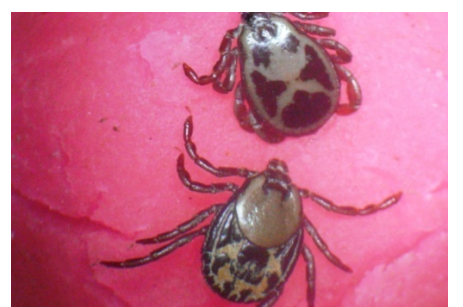

(b)

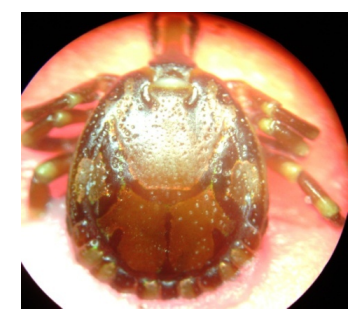

(c)

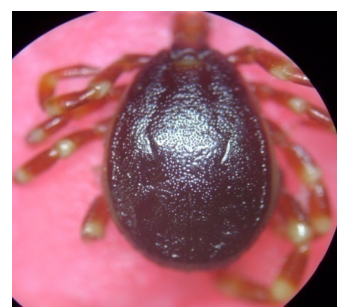

(d)

Plate 2. Ticks identified from Somali goats in Benadir area. (a) Rhipicephalus evertsi; (b) Rhipicephalus pulchellus; (c) Amblyomma lepidum; (d) Hyalomma rufipes. 
Ahmed A. Ismail, SUST and VRI for their technical support.

\section{REFERENCES}

[1] E. J. L. Soulsby, "Helminths, Arthropods \& Protozoa of Domesticated Animals," 7th Edition, Bailliere Tindall, London, 1986.

[2] G. M. Urquhart, J. Armour, J. L. Duncan, A. M. Dunn and F. W. Jennings, "Veterinary Parasitology," 2nd Edition, B-Blackwell Science, 1996.

[3] K. T. Friedhoff, "Transmission of Babesia," In: M. Ristic, Ed., Babesiosis of Domestic Animals and Man, CRC Press, Boca Raton, 1988, pp. 23-52.

[4] A. M. El Hussein, A. M. Majid and S. M. Hassan, "The
Present Status of Tick-Borne Diseases in the Sudan," Archives de l'Institut Pasteur de Tunis, Vol. 81, No. 5, 2004, pp. 31-34.

[5] S. M. Hassan and D. A. Salih, "Bibliography with Abstracts, Ticks and Tick-borne Diseases in the Sudan," 1st Edition, Central Laboratory, Ministry of Science and technology, Khartoum, 2009.

[6] FAO/World Bank Cooperative Programme, the World Bank, European Union Report No. 04/001 IC-SOM, 2004.

[7] Food and Agriculture Organization (FAO) of the United Nations investment centre division, Somalia, "Somalia towards a Livestock Sector Strategy,” Final Report, 1998.

[8] Food Security Assessment Unit (FSAU), 1999. 